\title{
SUNFLOWER SEED HUSK AGAR: A NEW MEDIUM FOR THE DIFFERENTIATION OF CANDIDA DUBLINIENSIS FROM CANDIDA ALBICANS
}

\author{
*ZU Khan, S Ahmad, E Mokaddas, N Al-Sweih, R Chandy
}

\begin{abstract}
A sunflower (Helianthus annuus) seed husk agar medium has been developed and evaluated for differentiation of Candida dubliniensis from Candida albicans on the basis of colony morphology and chlamydospore production. All C. dubliniensis isolates $(n=40)$ produced rough colonies with hyphal fringes and abundant chlamydospores whereas 101 of $105(96.2 \%)$ C. albicans isolates produced smooth colonies with no evidence of chlamydospore production. Since this medium is free from oil droplets, chlamydospores can be examined with greater clarity by Dalmau plate technique. This medium provides a simple and cost-effective tool for the presumptive differentiation of C. dubliniensis from C. albicans and is particularly suited for clinical microbiology laboratories where biochemical or molecular methods for the differentiation of these two species are not available.
\end{abstract}

Key words: Sunflower, C. dubliniensis, C. albicans, rough colonies, chlamydospores

Candida dubliniensis is a newly described species of yeast and is phylogenetically closely related to Candida albicans. ${ }^{2}$ Although initially implicated in oral candidiasis in HIVinfected and AIDS patients, it is capable of causing a variety of clinical conditions including vaginal and bloodstream infections. ${ }^{3,4}$ Since $C$. dubliniensis and $C$. albicans have phenotypic similarities, such as ability to form germ tubes and chlamydospores, their differentiation in routine clinical microbiology laboratories is problematic and may lead to misidentification. ${ }^{5}$ During the last five years several tests based on phenotypic characteristics have been developed to distinguish these two species. ${ }^{6-11}$ In 1999, Staib and Morschausser ${ }^{6}$ extended the diagnostic usefulness of Staib's bird seed (Guizotia abyssinica) agar, ${ }^{12}$ originally used as a differential medium for Cryptococcus neoformans, to distinguish $C$. dubliniensis from $C$. albicans. Candida dubliniensis isolates formed rough colonies with abundant chlamydospores on this medium, whereas $C$. albicans isolates formed smooth colonies with no chlamydospore production. These observations were subsequently confirmed by AlMosaid et al. ${ }^{7}$ and Lees and Barton. ${ }^{8}$ Recently, sunflower (Helianthus annuus) seed agar has also been found useful for distinguishing $C$. dubliniensis from $C$. albicans on the basis of similar morphologic characteristics as have been observed on niger seed agar. ${ }^{9-11}$ In this communication, we report that husk of sunflower seeds can be used as a substitute for whole seeds in the medium without compromising its efficacy.

\section{Materials and Methods}

Sunflower seed husk medium

* Corresponding author (email: <ziauddin@hsc.edu.kw>) Department of Microbiology, Faculty of Medicine, Kuwait University, PO Box 24923, Kuwait - 13110

Received: 19-11-2004

Accepted: 21-2-2005
The sunflower seeds were purchased from open market and the husk was separated manually. The medium was prepared as follows: 50 gram sunflower seed husk was pulverized in domestic grinder for 3-4 minutes. The pulverized husk was boiled for 30 minutes with 1 litre of distilled water and filtered through several layers of gauze. To husk extract so obtained were added 15 gram agar (Difco Laboratories, Detroit, USA) and 10 gram glucose. The volume was made up to 1 litre and $\mathrm{pH}$ adjusted to 5.5 before autoclaving at 15 pounds pressure for 15 minutes.

\section{Reference strains and test cultures}

Two reference strains (CBS 7987 and CD 36) and 38 local clinical isolates of $C$. dubliniensis (sputum-17, vagina- 8 , endotracheal secretions-8, urine-3, and one each from bronchoalveolar lavage and catheter tip), and three reference strains (ATCC 90029, ATCC 2091, ATCC 90028) and 102 clinical isolates of Candida albicans were included in the study. All the reference strains and test isolates were freshly subcultured and tested for germ tube formation in pooled human serum and for chlamydospore production on cornmeal agar (Becton Dickinson, France) supplemented with $1 \%$ Tween 80 (CM-T) by Dalmau plate technique. ${ }^{13}$ Their identity was further confirmed by Vitek 2 ID-YST card and/or ID 32C system (bioMerieux, France) as well as by semi-nested PCR (snPCR) using species-specific primers corresponding to unique sequences within the internally transcribed spacer 2 (ITS 2) of $C$. dubliniensis and C. albicans and/or by direct sequencing of ITS 2. ${ }^{14,15}$

\section{Dalmau plate culture}

All the isolates forming germ tubes and chlamydospores on CM-T were streaked on sunflower husk (SSH) agar for studying colony morphology and chlamydospore production. Using a marker pen, the plate was divided into two halves, 
and each half on the bottom of the plate was labeled with the culture number. With a sterile inoculating needle, two streaks, approximately $1.5 \mathrm{~cm}$ long, were made on the plate one centimetre apart without digging into the medium. Only a very small quantity of the culture was streaked. After flaming and cooling the needle, an S-shaped streak was made across the two streaks made earlier. Coverslip (22 X $22 \mathrm{~mm})$ was flame sterilized, cooled and placed over the streak marks. The plates were incubated at $28^{\circ} \mathrm{C}$ and examined under microscope using low $(10 \mathrm{X})$ and high power $(40 \mathrm{X})$ at 24,48 and $72 \mathrm{~h}$ intervals. All the isolates showing fringed colonies with chlamydospores were re-examined for reproducibility by directly inoculating SSH plates by streaking.

\section{Results}

The observations on colonial morphology and chlamydospore formation by $C$. dubliniensis and $C$. albicans on SSH agar and CM-T agar are presented in the table and depicted in figures 1-3. Dalmau plate cultures of all the 40 C. dubliniensis isolates including two reference strains produced rough colonies with hyphal fringes and abundant chlamydospores on SSH agar after 24 to 48 hours of incubation at $28^{\circ} \mathrm{C}$. In contrast, $101(96.2 \%)$ C. albicans isolates including three reference strains produced smooth colonies with no evidence of chlamydospore formation on this medium. Of the four isolates which showed fringed colonies, only two produced scanty chlamydospores. The results were reproducible when the experiment was repeated. On CM-T agar, all the $C$. dubliniensis isolates and $C$. albicans isolates produced smooth colonies and chlamydospores, and no discernible differences were observed in the colony morphology of the two species.

\section{Discussion}

The phenotypic characteristics of $C$. albicans to form

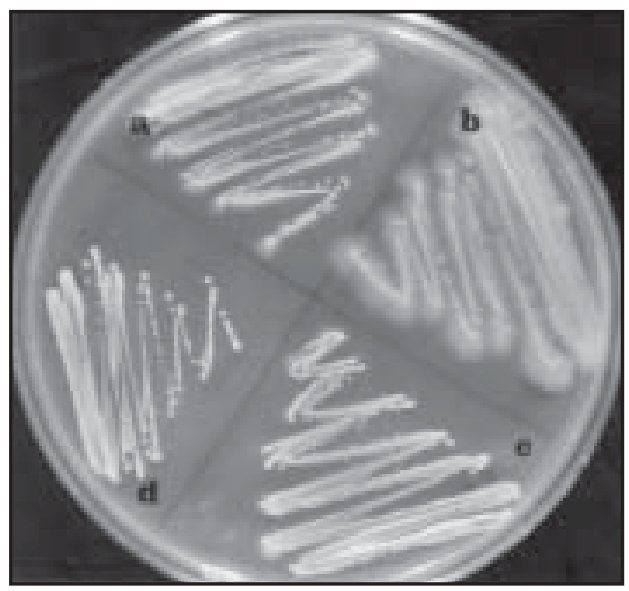

Figure 1: Sunflower seed husk plate showing growth of three stains of C. dubliniensis (a-CBS 7987, b-848/02, c- 239/03) and a clinical isolate of $C$. albicans (d- 6/03) after 3 days of incubation at $28^{\circ} \mathrm{C}$. Note: rough colonies with hyphal fringes of $C$. dubliniensis and smooth colonies of C. albicans
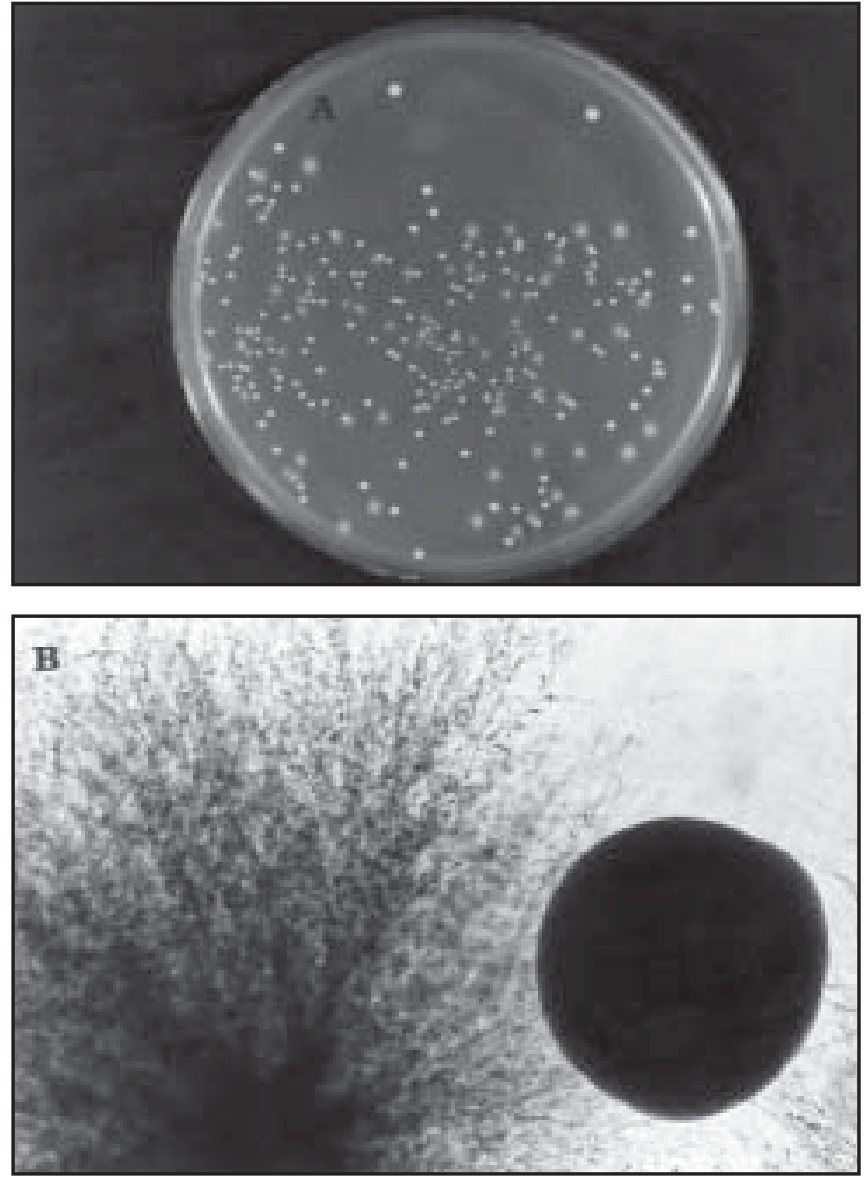

Figure 2: (A) Mixed culture of C. dubliniensis (CD-36, type strain) and C. albicans (ATCC 90028) on sunflower seed husk agar showing rough colonies with hyphal fringes of $C$. dubliniensis and small smooth colonies of $C$. albicans after 2 days of incubation at $28^{\circ} \mathrm{C}$. (B) microscopic view of a portion of $C$. dubliniensis colony showing hyphae with chlamydospores (left side), and a C. albicans colony with entire margin (right side). Magnification x 100.

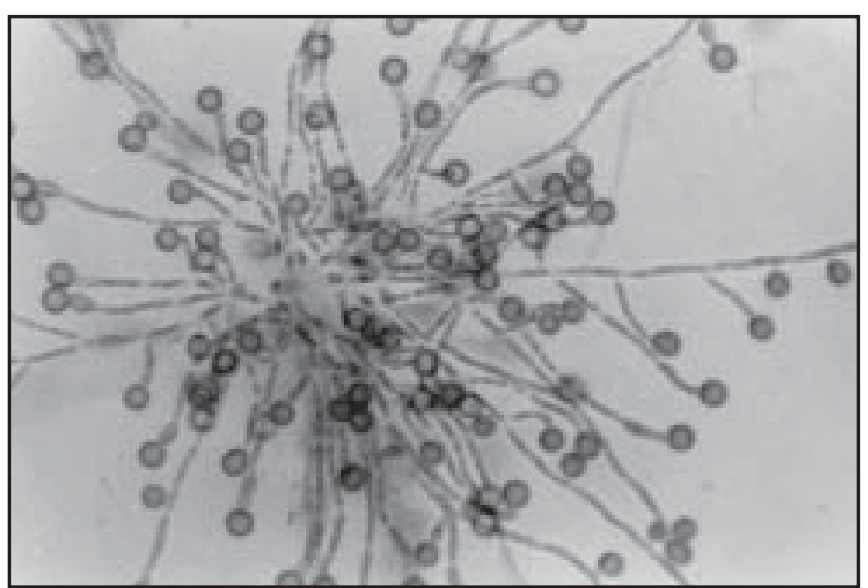

Figure 3: Dalmau plate culture on SSH agar showing chlamydospores of C. dubliniensis, strain 848/02, (magnification x 400).

chlamydospores and germ tubes have long been used to differentiate it from other Candida species. However, with the recognition of $C$. dubliniensis as a separate species, ${ }^{2,3}$ which 
Table: Observations on colony morphology and chlamydospore production on sunflower seed husk (SSH) agar and cornmeal-Tween 80 (CM-T) agar by $C$. dubliniensis and C. albicans

\begin{tabular}{lccccc}
\hline $\begin{array}{l}\text { Morphologic } \\
\text { characteristics }\end{array}$ & \multicolumn{2}{c}{ C. dubliniensis } & & \multicolumn{2}{c}{ C. albicans } \\
\cline { 2 - 3 } \cline { 5 - 6 } $\begin{array}{l}\text { Rough colonies with } \\
\text { hyphal fringes }\end{array}$ & SSH agar & CM-T agar & & SSH agar & CM-T agar \\
$\begin{array}{l}\text { Chlamydospore } \\
\text { production }\end{array}$ & $40 / 40 *(100 \%)$ & $0 / 40(0 \%)$ & & $4 / 105(3.8 \%)$ & $0 / 105(0 \%)$ \\
\hline
\end{tabular}

*Number positive/number tested, ${ }^{\text {a }}$ showed only scanty chlamydospores

like C. albicans produces chlamydospores and germ tubes, several investigators have focused attention to develop simple and inexpensive methods for discriminating these two species in routine clinical microbiology laboratories. The tests that have been reported to be useful for this purpose include production of rough colonies and chlamydospores on Staib agar, ${ }^{6-8}$ development of dark green colonies on CHROMagar Candida, ${ }^{2,16}$ inability to grow at $45{ }^{\circ} \mathrm{C},{ }^{17}$ and in $6.5 \%$ sodium chloride $^{18}$ by $C$. dubliniensis isolates. Recently, several commercial yeast identification systems based on carbohydrate assimilation profiles have also become available for the identification of $C$. dubliniensis. ${ }^{3,4}$ In an extensive evaluation of commercial yeast identification systems which included API 20C AUX, ID 32 C, RapID yeast plus, Vitek YBC, and Vitek 2 ID YST, Pincus et al. ${ }^{19}$ reported that assimilation of a-methyl-D-glucoside, trehalose, and D-xylose could be used for the differentiation of $C$. dubliniensis. However, the assimilation results were not consistent with different commercial assimilation systems used and varied with the incubation period..$^{19,20}$

Several investigators have reported that $C$. dubliniensis isolates have the ability to produce chlamydospores more readily and abundantly than $C$. albicans on rice agar-Tween, Tween 80-oxgall-caffeic acid agar or cornmeal-Tween 80 $\operatorname{agar}^{2,3}$ and this morphologic characteristic could be used for differentiating the two species. However, identification of $C$. dubliniensis on the basis of abundance of chlamydospores or other phenotypic features have not been shown to be reproducible in some laboratories. ${ }^{16}$ Moreover, assessment of relative abundance of chlamydospores is quite subjective in judgment, hence this feature alone may not serve as a dependable criterion for differentiation of $C$. dubliniensis from C. albicans. Recently, Staib and Morschhauser ${ }^{6}$ reported that C. dubliniensis on Staib's G. abyssinca seed agar ${ }^{12}$ under appropriate growth conditions forms rough colonies due to mycelial growth and abundant chlamydospores which may be used as a species-specific marker for identification of this species. In an extended evaluation of this medium, Al-Mosaid et $a l^{7}$ found that $85.4 \%$ of the $C$. dubliniensis isolates ( $\mathrm{n}=$ 130) while none of the $C$. albicans isolates $(n=166)$ produced chlamydospores on this medium. In contrast, while all the isolates of $C$. albicans produced smooth colonies, as many as $97.7 \%$ isolates of $C$. dubliniensis formed rough colonies. The authors concluded that morphological discrimination between the two species is best achieved on the basis of colony morphology rather than chlamydospore production. In a more recent study, Adou-Bryn et $a l^{9}$ using a modified sunflower seed agar observed that all the $60 \mathrm{C}$. dubliniensis isolates produced rough colonies, whereas all the $47 C$. albicans isolates produced smooth colonies. Efficacy of sunflower seed agar was subsequently confirmed by AlMosaid et $a l^{10}$ and Khan et al. ${ }^{11}$

In conclusion, production of rough colonies and abundant chlamydospores by all the 40 isolates of $C$. dubliniensis, and of smooth colonies with lack of chlamydospores by over $96 \%$ isolates of $C$. albicans, suggests that $\mathrm{SSH}$ agar has a value in the presumptive differentiation of these two clinically important species. The low production cost, wider availability of the ingredients and simple composition make SSH medium ideally suited for routine use in clinical microbiology laboratories. Since the medium is free from oil droplets, it provides a clearer view of chlamydospores during microscopic examination, a problem that could be encountered with media prepared from the whole seeds.

\section{Acknowledgments}

Supported by Kuwait University Research Administration grant MI 118.

\section{References}

1. Sullivan DJ, Westerneng TJ, Haynes KA, Bennet DE, Coleman, D. Candida dubliniensis sp. nov., phenotypic and molecular characterization of a novel species associated with oral candidiasis in HIV-infected individuals. Microbiol 1995; 141:1507-21.

2. Sullivan D, Coleman, D. Candida dubliniensis, characteristics and identification. J Clin Microbiol 1998;36:329-34.

3. Gutierrez J, Morales P, Gonzalez MA, Quindos G. Candida dubliniensis, a new fungal pathogen. J Basic Microbiol 2002; 3:207-27. 
4. Sullivan DJ, Moran GP, Pinjon E, Al-Mosaid A, Stokes C, Vaughan $\mathrm{C}$, et al. Comparison of the epidemiology, drug resistance mechanisms, and virulence of Candida dubliniensis and Candida albicans. FEMS Yeast Res 2004;4:369-76.

5. Odds FC, Van Nuffel L, Dams G. Prevalence of Candida dubliniensis isolates in a yeast stock collection. J Clin Microbiol 1998;36:2869-73.

6. Staib P, Morschhauser J. Chlamydospore formation on Staib agar as a species-specific characteristic of Candida dubliniensis. Mycoses 1999;42:521-4.

7. Al-Mosaid, A., Sullivan, D., Salkin, I. F., Shanley, D. and Coleman, D.C. Differentiation of Candida dubliniensis from Candida albicans on Staib agar and caffeic acid-citrate agar. $J$ Clin Microbiol 2001;39:323-7.

8. Lees E, Barton RC. The use of Niger seed agar to screen for Candida dubliniensis in the clinical microbiology laboratory. Diagn Microbiol Infect Dis 2003;46:13-7.

9. Adou-Bryn K, Douchet C, Ferrer A, Grimaud L, Robert R, Richard-Lenoble D. Morphologic features of Candida dubliniensis on a modified Pal's medium. Preliminary study. $J$ Mycol Med 2003;13:99-103.

10. Al-Mosaid A, Sullivan DJ, Coleman DC. Differentiation of Candida dubliniensis from Candida albicans on Pal's agar. $J$ Clin Microbiol 2003;41:4787-9.

11. Khan ZU, Ahmad S, Mokaddas E, Chandy R. Simplified sunflower (Helianthus annuus) seed agar for differentiation of Candida dubliniensis from Candida albicans. Clin Microbiol Infect 2004;10:590-2.

12. Staib F, Seibold M, Antweiler E, Frohlich B, Weber S, Blisse A. The brown colour effect (BCE) of Cryptococcus neoformans in the diagnosis, control and epidemiology of $C$. neoformans infections in AIDS patients. Zentralbl Bakteriol Mikrobiol Hyg.
[A]. 1987;266:167-77.

13. McGinnis, R. Laboratory handbook of medical mycology, Academic Press, New York. 1980.

14. Ahmad S, Khan Z, Mustafa AS. Khan ZU. Seminested PCR in the diagnosis of candidemia: comparison with culture, antigen detection, and biochemical methods for identification. $J$ Clin Microbiol 2002;40:2483-9.

15. Ahmad S, Khan Z, Mokaddas E. Khan ZU. Isolation and molecular identification of Candida dubliniensis from nonhuman immunodeficiency virus-infected patients in Kuwait. $J$ Med Microbiol 2004;56:633-7.

16. Schoofs A, Odds FC, Colebunders R, Leven M, Goosen H. Use of specialized isolation media for recognition and identification of Candida dubliniensis isolated from HIV-infected patients. Eur J Clin Microbiol Infect Dis 1997;16:296-300.

17. Pinjon E, Sullivan D, Salkin I, Shanley D, Coleman, DC. Simple, inexpensive, reliable, method for differentiation of Candida dubliniensis from Candida albicans. J Clin Microbiol 1998;36:2093-5.

18. Alves SH, Milan EP, Ana Pde LS, Oliveria LO, Santurio JM, Colombo AL. Hypertonic Sabouraud broth as a simple and powerful test for Candida dubliniensis screening. Diag Mirobiol Infect Dis 2002;43:85-6.

19. Pincus DH, Coleman DC, Pruitt WR, Padhey AA, Salkin IF, Geimer M, et al. Rapid identification of Candida dubliniensis with commercial yeast identification systems. J Clin Microbiol 1999;37:3533-9.

20. Gales AC, Pfaller MA, Houston AK, Jolly S, Sullivan DJ, Coleman DC, Soll DR. Identification of Candida dubliniensis based on temperature and utilization of xylose and alpha-methylD-glucoside as determined with the API 20C AUX and Vitek YBC Systems. J Clin Microbiol 1999;37:3804-8. 